\title{
Non-invasive prediction of lymph node status for patients with early-stage invasive breast cancer based on a morphological feature from ultrasound images
}

\author{
Tao Jiang ${ }^{\#}$, Weiwei Su${ }^{\#}$, Yanan Zhao^, Qunying Li, Pintong Huang \\ Department of Ultrasound, The Second Affiliated Hospital of Zhejiang University School of Medicine, Hangzhou, China
}

Contributions: (I) Conception and design: T Jiang, W Su; (II) Administrative support: P Huang; (III) Provision of study materials or patients: W Su; (IV) Collection and assembly of data: W Su, Y Zhao, Q Li; (V) Data analysis and interpretation: T Jiang; (VI) Manuscript writing: All authors; (VII) Final approval of manuscript: All authors.

"These authors contributed equally to this work.

Correspondence to: Pintong Huang. Department of Ultrasound in Medicine, The Second Affiliated Hospital of Zhejiang University School of Medicine, Zhejiang University, 88 Jiefang Road, Shangcheng District, Hangzhou 310009, China. Email: huangpintong@zju.edu.cn.

\begin{abstract}
Background: This study aimed to estimate the value of a morphological feature on ultrasound (US) for preoperative diagnosis of axillary lymph node (ALN) status in patients with early-stage invasive breast cancer (ESIBC).

Methods: In this retrospective work, a total of 239 ESIBC patients, were recruited, and their preoperative US images and postoperative pathology results were collected. The relationship between US images based on morphological features and ALN metastasis was investigated. The tumor circularity and US-reported ALN status were developed as a nomogram to predict the ALN status.

Results: Among the 239 participants, 82 (34.31\%) had ALN metastasis, and 157 (65.69\%) did not. There was a statistically significant difference in tumors between participants diagnosed with and without ALN metastasis. The median value was 0.47 vs. $0.62(\mathrm{P}<0.001)$ in the training group, respectively, and the value was 0.50 vs. $0.60(\mathrm{P}<0.001)$ in the validation group, respectively. The clinical model nomogram was shown to have high efficiency in predicting ALN status among our research population. The area under the curve (AUC) was 0.89 in the training group and 0.90 in the validation group and the accuracy was $85.79 \%$ and $81.63 \%$, respectively.
\end{abstract}

Conclusions: The clinical model nomogram based on tumor circularity and US-reported ALN status is a non-invasive approach for ALN metastasis prediction in ESIBC patients with high efficacy.

Keywords: Breast neoplasms; lymphatic metastasis; tumor shape; ultrasound

Submitted Oct 25, 2020. Accepted for publication Apr 01, 2021.

doi: 10.21037/qims-20-1201

View this article at: http://dx.doi.org/10.21037/qims-20-1201

\section{Introduction}

Axillary lymph node (ALN) status plays an important role in the staging, therapy, and prognosis of early-stage invasive breast cancer (ESIBC) patients who have the clinically negative axillary disease (1-3). Previously, ALN dissection (ALND) was the conventional method for determining ALN status, and it was accompanied by radical mastectomy

^ ORCID: Tao Jiang, 0000-0002-3690-6678; Weiwei Su, 0000-0001-7870-7813; Yanan Zhao, 0000-0002-4938-9235. 
for local disease control. In the past few years, sentinel node biopsy (SNB) has developed as an alternative to ALND and has become the regular treatment for axillary evaluation in clinically negative patients (4-6). The SNB is a minimally invasive method and has a significantly lower incidence rate of complications such as lymphedema, numbness, pain, and shoulder impairment than ALND $(7,8)$. However, clinical studies have indicated that SNB may also cause some complications like wound infection, hematoma, and abnormal sensation (9). Sometimes, a biopsy may be difficult and risky when lymph nodes are adjacent to blood vessels or positioned deeply in the axilla. Furthermore, the false-negative rate of SNB cannot be overlooked, ranging from $5 \%$ to $10 \%(10,11)$, and may lead to a second surgery and increase in cost. Therefore, ALN differentiation remains clinically significant, and a non-invasive and reliable approach is urgently required to evaluate ALN status without the use of SLNB in patients without ALN metastasis.

Ultrasound (US) examination is a convenient, noninvasive, and inexpensive approach for assessing breast tumors. Axillary US examination is also a useful method for assessing ALN status by using the characteristics of the lymph node morphologic features, size, cortical thickness, lymphoid hilar structure, blood flow, and boundary (12-14). However, US-image-based breast tumors and ALN assessments rely on macroscopic appearances in grayscale visible to the naked eye. These image features are subjective and depend on the experience of the interpreters.

Radiomics is a method of transforming digital medical images into high-dimensional data then extracting numerous quantitative features to improve diagnosis, prognosis, and prediction accuracy. Previous studies have suggested that the US, magnetic resonance imaging (MRI), and mammographybased radiomics analysis are reliable and non-invasive tools for assessing ALN status in patients with ESIBC (15-17). However, radiomics analysis of US images still lacks reproducibility. This means that we need a stable and unbiased feature from US images to predict ALN status.

In this work, we developed and validated a US-imagebased nomogram to predict the probability of ALN metastasis in ESIBC patients.

\section{Methods}

\section{Patients}

The study was conducted in accordance with the Declaration of Helsinki (as revised in 2013). Our Institutional Review
Board approved the study, and as retrospective research, informed consent was waived. A total of 239 women with ESIBC who underwent breast surgery with ALN evaluation between 1 January 2018 and 31 January 2020 at our hospital were included in this study. The clinical data and US images were obtained from our hospital's PACS system.

The inclusion criteria were as follows: (I) patients with clinical T1or T2 primary invasive breast carcinoma confirmed by resection or biopsy, no palpable axillary mass; (II) patients present with only 1 solid tumor; (III) no neoadjuvant chemotherapy (NAC) before US examination; (IV) US examinations obtained within 2 weeks before surgery or biopsy; (V) acceptable high-quality US image of ALN examination. The exclusion criteria were as follows: (I) breast tumor on the US image was not completely visible; (II) incomplete clinical data; (III) pathological result of ALN status was missing. The research population recruitment pathway is presented in Figure 1. We selected 30 June 2019 as the cutoff point to split the population into training and time-independent test cohort with a 4:1 ratio, as previously described (18). Finally, 190 patients who underwent surgery between 1 January 2018, and 30 June 2019, were included as the training group, and 49 patients who were treated between 1 July 2019, and 31 January 2020, were included as the validation group.

\section{Clinical data}

The clinical features were retrieved from the PACS system, including age, lymph node status, and histologic tumor type.

\section{B-Mode US examinations and tumor morphologic features}

Experienced examiners performed preoperative US examinations at our department. During the exam, the participant was placed in a supine position with both hands behind the head to expose the breasts and axilla fully. As a retrospective analysis, the US images were received from various US systems, including AixPlorer (SuperSonic Imagine, Aix-en-Province, France), Acuson S3000 (Siemens Medical Solutions, Mountain View, CA, USA), MyLab Twice (Esaote, Genoa, Italy), Resona 7 (Mindray, Shenzhen, China), and the probe frequency ranged from 5 to $13 \mathrm{MHz}$.

For the target tumor, images of the best quality were selected from the PACS system for our study. The largest diameter, which measured in the most significant slice of the tumor, was defined as tumor size, the region of interest 


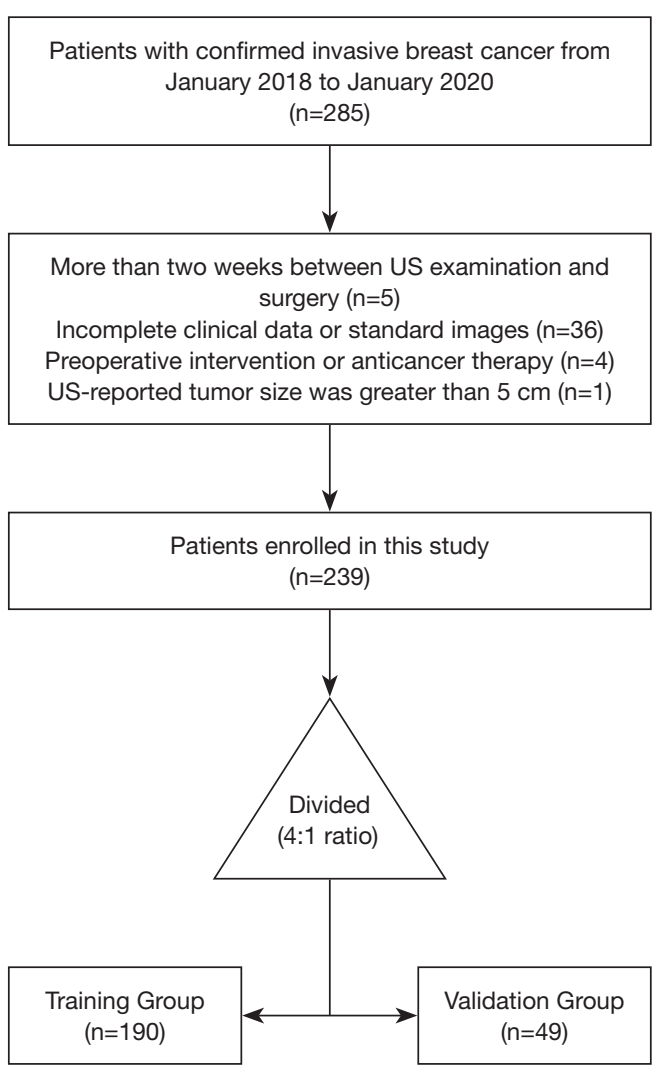

Figure 1 Recruitment pathway for participant selection.

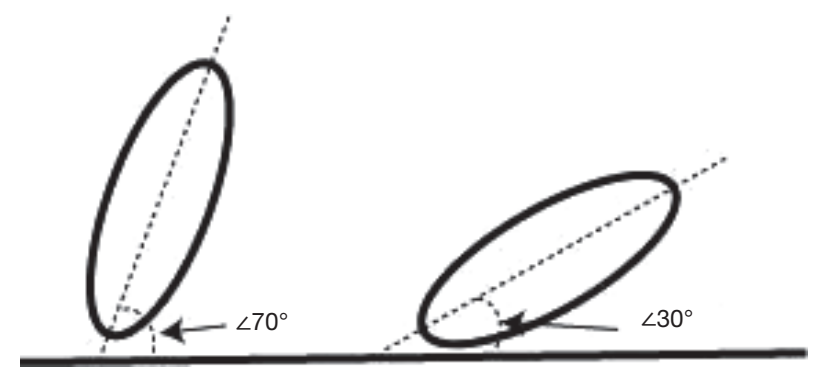

Figure 2 The relationship between orientation and tumor aspect ratio.

(ROI) covering the tumor was manually delineated by an experienced radiologist using the commercial software MatLab (version 2019, MathWorks, Natick, MA, USA) on the grayscale US image. The circularity, eccentricity, and orientation parameters were directly calculated with the MatLab tool "regionprops."

\section{Circularity}

The circularity parameter was calculated as $\left(4^{*} \mathrm{Area}{ }^{*} \mathrm{pi}\right) /$ $\left(\right.$ Perimeter $\left.^{2}\right)$. It specified the roundness of the tumor; for a perfect circle, the circularity value was 1 . The circularity value was close to 0 if a tumor was very irregular in shape.

\section{Eccentricity}

This parameter was defined as the ratio of the distance between the foci of the ellipse and its primary axis length. The value of this feature was between 0 and 1 . The eccentricity value was 0 if a tumor shape was a circle, and the value close to 1 if it's like a line segment.

\section{Orientation}

Orientation was defined as the angle between the $\mathrm{x}$-axis and the ellipse's major axis that had the same second-moments as the region. The value of this parameter was in degrees and ranged from $-90^{\circ}$ to $90^{\circ}$. The axes and orientation of the ellipse are illustrated in Figure 2. We were able to quickly deduce that if the absolute value of orientation is greater than $45^{\circ}$, the tumor aspect ratio was $>1$.

\section{ALN status}

The ALN status was assessed with morphologic features, cortical thickness, and lymphoid hilar structure of the lymph node. A US-reported positive ALN status suspicious of metastasis was defined as the longest/shortest axes ratio $<2$, irregular cortical thickness greater than $3 \mathrm{~mm}$, or absence of fatty hilum $(12,16,19)$. The axillary node images in the database were independently evaluated by 2 authors involved in this study without them knowing the details of clinical and pathological data. If the results were different, the final outputs were decided via discussion with a senior doctor (Pintong Huang, with 20 years' experience).

\section{Statistical analysis}

The R software package (V3.6.3, https://www.R-project. org/) was used for statistical analysis. Numeric data of all participants were expressed as mean \pm standard deviation (SD). The descriptive statistics were divided into categorical and continuous variables. We used Pearson's chi-square or Fisher's exact test to compare differences in categorical variables. Continuous variables were analyzed by nonparametric Mann-Whitney $U$ test or Student $t$-tests. Multivariate logistic regression analysis was introduced to determine the optimum features that were related to ALN metastasis. A P value $<0.05$ was considered statistically significant. 
Table 1 Clinical characteristics of the training and the validation group

\begin{tabular}{|c|c|c|c|c|c|c|}
\hline Characteristic & \multicolumn{3}{|c|}{ Training group $(n=190)$} & \multicolumn{3}{|c|}{ Validation group $(\mathrm{n}=49)$} \\
\hline Age (y) & $54.91(9.54)$ & $55.89(9.91)$ & 0.449 & 56.67 (9.73) & 56.89 (9.65) & 0.690 \\
\hline Size (cm) & $1.98(0.91)$ & $2.23(0.80)$ & 0.049 & $2.10(0.86)$ & $2.23(0.93)$ & 0.910 \\
\hline Eccentricity & $0.72(0.14)$ & $0.73(0.15)$ & 0.255 & $0.71(0.14)$ & $0.68(0.20)$ & 0.943 \\
\hline \multicolumn{2}{|c|}{ Internal microcalcification } & & 0.024 & & & 0.163 \\
\hline Yes & 72 & 24 & & 20 & 8 & \\
\hline LN-negative & 114 & 17 & & 27 & 6 & \\
\hline Histologic type & & & 0.081 & & & 0.103 \\
\hline Ductal & 67 & 30 & & 13 & 10 & \\
\hline Lobular or mixed & 49 & 32 & & 9 & 1 & \\
\hline Other & 11 & 1 & & 8 & 8 & \\
\hline
\end{tabular}

Data are shown as mean \pm SD. LN, lymph node; ALN, axillary lymph node; US, ultrasound.

To evaluate the intra-observer and inter-observer reproducibility of the parameters, we randomly chose additional 30 tumor images, 2 independent radiologists completed ROI segmentation, and 1 operator repeated the work 2 weeks later. The interclass correlation coefficients (ICCs) values were calculated. The parameters extraction had good repeatability if the ICCs values were greater than 0.80 .

\section{Results}

After screening, there were 239 patients included in this study. Participant clinical characteristics in the training and validation groups are presented in Table 1. Participants with ALN metastases formed $33.16 \%$ (63/190) of the training group and $38.78 \%(19 / 49)$ of the validation group. The sensitivity and specificity of the US-reported ALN status in the training group and the validation group were $73.02 \%$ $(46 / 63)$ and $89.76 \%(114 / 127)$, and $68.42 \%(13 / 19)$ and $90.00 \%$ (27/30), respectively. Positive predictive values and negative predictive values were $77.97 \%(46 / 59)$ and $87.02 \%(114 / 131)$, respectively, in the training group, and
$81.25(13 / 16)$ and $81.82 \%(27 / 33)$ in the validation group, respectively.

The intra-observer ICCs value was 0.93 , and the values ranged from 0.89 to 0.91 in inter-observer ICCs. These results indicated that the parameters in this work had good repeatability and stability.

There was a considerable difference in the tumor size parameter between participants with and those without ALN metastasis in the training group ( $\mathrm{P}=0.049)$. Meanwhile, in the validation group, the difference was not statistically significant $(\mathrm{P}=0.910)$.

Both in the training and validation groups, there was a statistically significant difference in tumor circularity between participants diagnosed with and without ALN metastasis. The median value was 0.47 vs. $0.62(\mathrm{P}<0.001)$ in the training group and the value was 0.50 vs. $0.60(\mathrm{P}<0.001)$ in the validation group.

The tumor internal microcalcification parameter was statistically significant in the training group $(\mathrm{P}=0.024)$ but not in the validation group $(\mathrm{P}=0.163)$.

Using multivariable regression analysis, tumor circularity 
Table 2 The results of the multivariable regression analysis

\begin{tabular}{|c|c|c|c|c|c|c|c|}
\hline Characteristic & \multicolumn{3}{|c|}{ Nomogram } & AUC & Sensitivity & Specificity & Accuracy \\
\hline Circularity & -6.381 & $0.224(0.112$ to 0.447$)$ & $<0.001$ & & & & \\
\hline US-ALN status* & 3.136 & 23.019 (9.373 to 56.531$)$ & $<0.001$ & & & & \\
\hline Training group & & & & $0.89(0.84-0.94)$ & $74.60 \%(47 / 63)$ & $91.34 \%(116 / 127)$ & $85.79 \%(163 / 190)$ \\
\hline
\end{tabular}

*US-ALN status is US reported ALN status. OR, odds ratio; Cl, confidence interval; US-ALN, ultrasound-axillary lymph node; AUC, area under the curve.

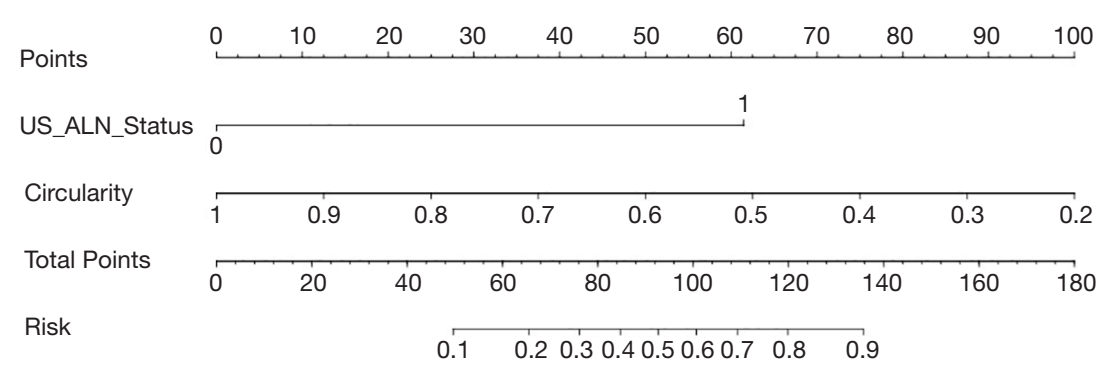

Figure 3 The clinical model nomogram developed with tumor circularity and US-reported ALN status for the prediction of ALN status. US, ultrasound; ALN, axillary lymph node.

and US-reported ALN status were selected from the following features: tumor size, tumor microcalcification, tumor circularity, and US-reported ALN status ( $\mathrm{P}$ value: $0.875,0.395,<0.001$, and $<0.001$, respectively). Table 2 displays the multivariable regression analysis results with the chosen 2 parameters in the training group. The variance inflation factor (VIF) of the 2 parameters was 1.07-1.79, suggesting there was no multicollinearity. Our clinical model was developed as a nomogram (Figure 3) incorporating tumor circularity and US-reported ALN status.

The ROC curves are displayed in Figure 4A,B. The clinical model had the most outstanding discrimination in the training group, and the area under the curve (AUC) reached 0.89. The model also presented a similar AUC value of 0.90 in the validation group. The calibration curves (Figure 4C,D) demonstrated that the predicted and observed ALN metastasis had durable consistency in the training and validation groups. Using the Hosmer-Lemeshow test, we got a non-statistically significant $P$ value of 0.59 in the training group and 0.66 in the validation group, indicating that our model fits very well. Hence, our clinical model worked well in both the training group and the validation group.

To categorize the participants into high-risk and lowrisk ALN metastasis groups, based on the maximum Youden index, the nomogram scores' best cutoff value was 89 . In the training group, the sensitivity, specificity, and accuracy of the clinical model were $74.60 \%$ (47/63), 91.34\% (116/127), and $85.79 \%(163 / 190)$, respectively. In the validation group, also showed excellent prediction efficacy with an AUC of 0.90 (95\% CI: 0.80 to 0.99 ), and the sensitivity, specificity, and accuracy were $68.42 \%(13 / 19), 90.00$ (27/30), and $81.63 \%$ (40/49), respectively. The decision curve analysis (DCA) based upon tumor circularity, US-reported ALN status, and clinical model is demonstrated in Figure 5. When the threshold probability for a patient ranged from 0.2 to 0.9 , the clinical model had the most clinical usefulness for predicting ALN metastasis.

\section{Discussion}

This study was designed to assess the US use as a noninvasive and cheap method for preoperative diagnosis of ALN status in patients with ESIBC. The results showed that the tumor circularity and US-reported ALN status 
A

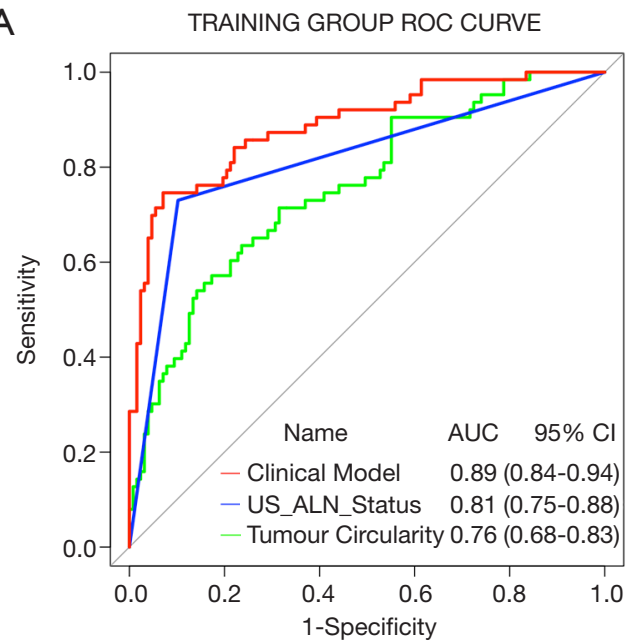

C

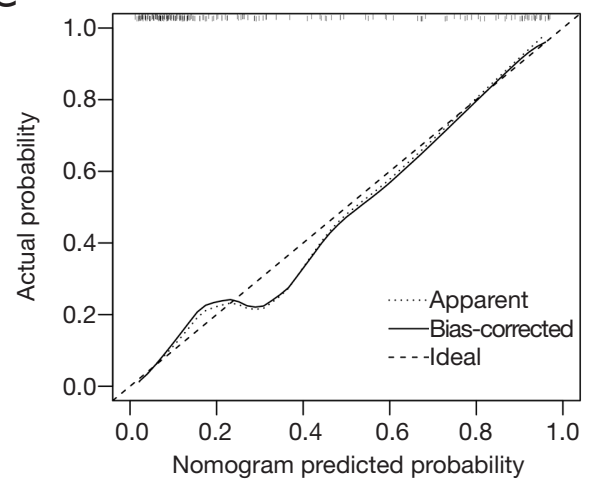

$B=1000$ repetitions, boot Mean absolute error $=0.022 n=190$
B

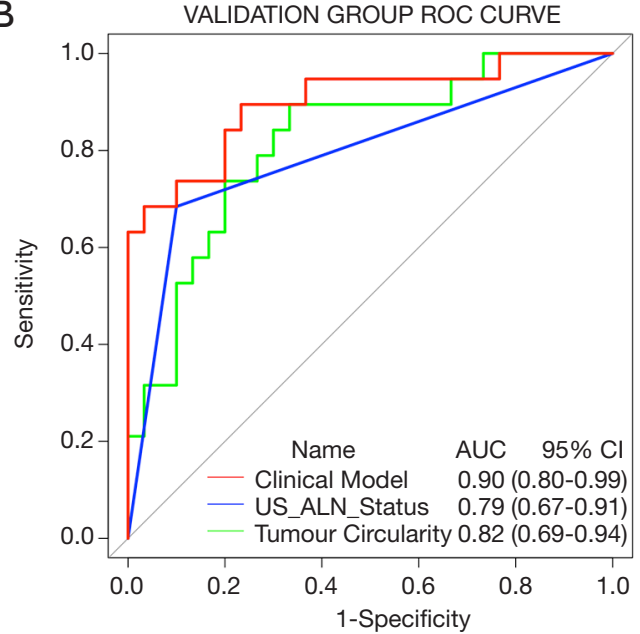

D

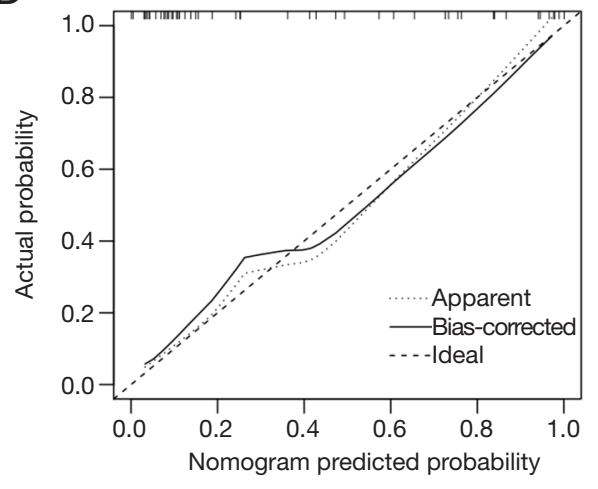

$B=1000$ repetitions, boot Mean absolute error $=0.034 n=49$

Figure 4 ROC curves and calibration curves. (A,B) The ROC curves of the training group and the validation group; (C,D) calibration curves of the nomogram in the training group and the validation group. ROC, receiver operating characteristic.

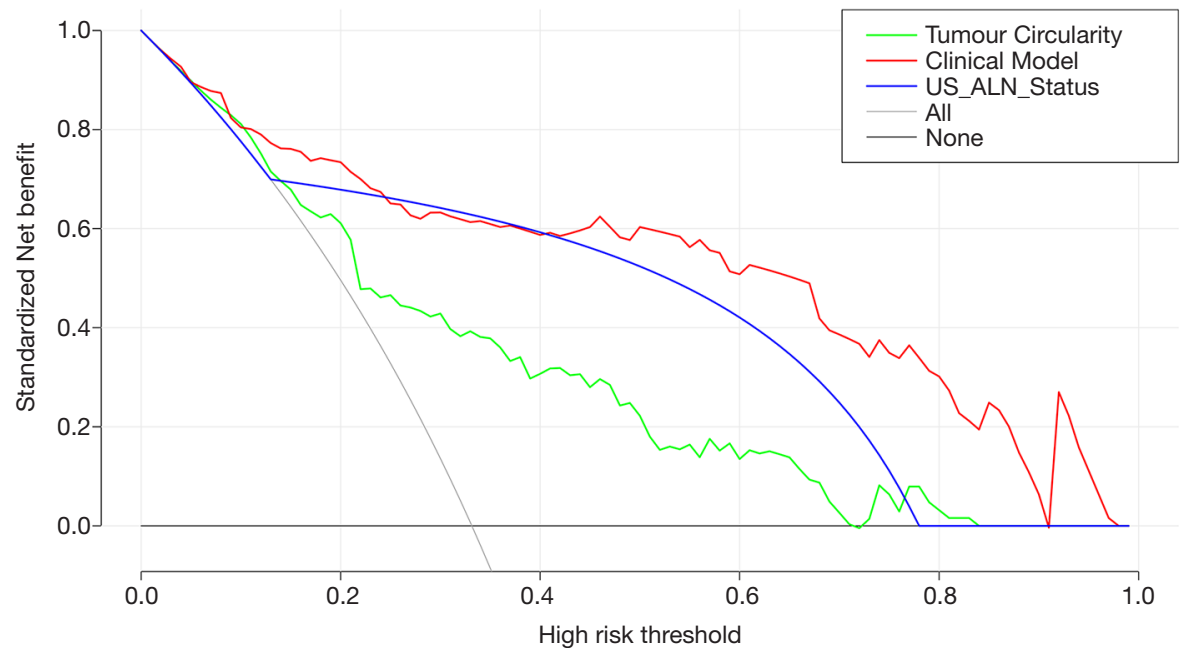

Figure 5 DCA for the clinical model in predicting ALN metastasis for patients with ESIBC. DCA, decision curve analysis; ALN, axillary lymph node; ESIBC, early-stage invasive breast cancer. 
were significantly associated with ALN metastasis. The clinical model nomogram based on circularity and USreported ALN status had high accuracy in predicting ALN status in our research population. The AUC was 0.89 in the training group and 0.90 in the validation group, and the accuracy was $85.79 \%$ and $81.63 \%$, respectively.

The ALN status plays an essential role in ESIBC patients' prognosis with clinically negative axillary disease $(6,20)$. Examination with the US is a convenient, noninvasive, and inexpensive method used extensively in diagnosing breast tumors and ALN evaluation. Recently, some researchers have explored the value of using the US features of primary breast tumors to predict ALN metastasis $(19,20)$. In their studies, tumor shape, tumor size, tumor doppler flow, and the tumor's distance from the nipple have been recognized as independent predictive factors for ALN metastasis. However, many of those factors are very subjective, especially tumor shape and doppler flow. The following non-invasive and preoperative parameters were analyzed in the current study to make our model more objective.

\section{Tumor size}

A commonly accepted theory is that as cancer expands, cells inside the tumor gain the capability to propagate, survive, and flourish within the regional lymph nodes. Consequently, in breast cancer patients, tumor size at diagnosis is linked to an increased likelihood of ALN metastasis. In our study, the result in the training group was similar to earlier research $(21,22)$, but in the validation group, the $\mathrm{P}$ value was higher than 0.05 , suggesting that there was no statistically significant difference in the tumor size between participants with and those without ALN metastasis in this group. There may be several reasons for this outcome: (I) the validation sample volume was tiny, and selection bias may have existed; (II) the connection between the tumor size and ALN metastasis may have been non-linearly correlated (21). Due to the $\mathrm{P}$ value of this parameter, it was not enrolled in the current study. The prognostic value of it is, however, still unclear, and further studies will be needed.

\section{Tumor circularity}

The tumor shape specifically reflects cancer growth characteristics. A benign tendency tumor usually has an expansive and slow-growing pattern, and as a result, the tumor will have a round shape. On the other hand, a tumor with an irregular shape typically represents an infiltrative growth model, which indicates fast-growth and a destructive tendency. Hence, the tumor shape characteristic indicates cancer cells' ability to invade the surrounding tissues (20). Tumor circularity is a quantitative parameter presented with an actual number and is an objective feature of tumor shape. Our study indicated that the circularity value was significantly associated with ALN metastasis both in the training and validation groups.

There are many available imaging methods for ALN assessment in patients diagnosed with ESIBC, including mammography, computed tomography, MRI (23), and US $(6,24)$. Compared with other imaging modalities, the axillary US exam is more convenient and effective $(6,25)$. The earlier study showed that by using the parameters of the ALN morphologic features, cortical thickness, blood flow, lymphoid hilar structure, and boundary to depict nonpalpable metastatic lymph nodes with the US, the sensitivity and specificity ranged from $26 \%$ to $76 \%$ and $88 \%$ to $98 \%$, respectively (26). The present work result was similar to the previous study, but our work's diagnostic criteria, such as L/S ratio, cortical thickness, and absence of fatty hilum, were quantitative characteristics.

Until now, there have been some nomograms established by clinical researches to predict ALN status $(9,17,27)$; but in several studies $(9,27)$, the variables included estrogen receptor (ER), progesterone receptor (PR), and tumor type, which can be obtained only via preoperative biopsy. Yu et al. (17) developed a US-based radiomics nomogram, and the AUC was 0.84 , but the radiomics research of grayscale US imaging still lacked reproducibility because of nonstandardized acoustic intensity and different US equipment with various settings. In our study, the tumor circularity was an objective parameter, and the variation between different equipment was rather small. Additionally, the clinical nomogram we developed used only 2 parameters that can be easily acquired preoperatively, and meanwhile, the AUC was similar to that of previous studies.

This retrospective study had some limitations. Firstly, our clinical model, which was established and validated for ALN status evaluation, was performed in a single hospital with limited sample size. Secondly, as retrospective research, it was difficult to identify which lymph nodes were biopsied or dissected and match the pathologic results to the images we analyzed. Thirdly, the images stored in our PACS system were acquired by different doctors of varied experience, 
resulting in selection bias.

\section{Conclusions}

This clinical model based on tumor circularity and USreported ALN status is a non-invasive approach for ALN metastasis prediction in ESIBC patients, and it can provide guidance and support for clinical activities.

\section{Acknowledgments}

Funding: This study was supported by the Natural Science Foundation of Zhejiang Province, China (LQ20H180009). The funders had no role in study design, data collection, analysis, decision to publish, or manuscript preparation.

\section{Footnote}

Conflicts of Interest: All authors have completed the ICMJE uniform disclosure form (available at http://dx.doi. org/10.21037/qims-20-1201). All authors reported that they receive grant from Natural Science Foundation of Zhejiang province, China (Grant No. LQ20H180009).

Ethical Statement: The authors are accountable for all aspects of the work in ensuring that questions related to the accuracy or integrity of any part of the work are appropriately investigated and resolved. The study was conducted in accordance with the Declaration of Helsinki (as revised in 2013). Our Institutional Review Board approved the study, and as retrospective research, informed consent was waived.

Open Access Statement: This is an Open Access article distributed in accordance with the Creative Commons Attribution-NonCommercial-NoDerivs 4.0 International License (CC BY-NC-ND 4.0), which permits the noncommercial replication and distribution of the article with the strict proviso that no changes or edits are made and the original work is properly cited (including links to both the formal publication through the relevant DOI and the license). See: https://creativecommons.org/licenses/by-nc-nd/4.0/.

\section{References}

1. Fusco R, Sansone M, Granata V, Di Bonito M, Avino F, Catalano O. Use of quantitative morphological and functional features for assessment of axillary lymph node in breast dynamic contrast-enhanced magnetic resonance imaging. Biomed Res Int 2018;2018:2610801.

2. Carter CL, Allen C, Henson DE. Relation of tumor size, lymph node status, and survival in 24,740 breast cancer cases. Cancer 1989;63:181.

3. Humphrey KL, Saksena MA, Freer PE. To do or not to do: axillary nodal evaluation after ACOSOG Z0011 Trial. Radiographics 2014;34:1807-16.

4. Lyman GH, Temin S, Edge SB, Newman LA, Turner RR, Weaver DL. Sentinel lymph node biopsy for patients with early-stage breast cancer: American Society of Clinical Oncology clinical practice guideline update. J Clin Oncol 2014;32:1365-83.

5. de Meric de Bellefon M, Lemanski C, Ducteil A, Fenoglietto P, Azria D, Bourgier C. Management of the Axilla in the Era of Breast Cancer Heterogeneity. Front Oncol 2018;8:84.

6. Marino MA, Avendano D, Zapata P, Riedl CC, Pinker K. Lymph Node Imaging in Patients with Primary Breast Cancer: Concurrent Diagnostic Tools. Oncologist 2020;25:e231-e242.

7. Qiu SQ, Zhang GJ, Jansen L, de Vries J, Schröder CP, de Vries EGE, van Dam GM. Evolution in sentinel lymph node biopsy in breast cancer. Crit Rev Oncol Hematol 2018;123:83-94.

8. Sun SX, Moseley TW, Kuerer HM, Yang WT. ImagingBased Approach to Axillary Lymph Node Staging and Sentinel Lymph Node Biopsy in Patients With Breast Cancer. AJR Am J Roentgenol 2020;214:249-58.

9. Chen JY, Chen JJ, Yang BL, Liu ZB, Huang XY, Liu GY. Predicting sentinel lymph node metastasis in a Chinese breast cancer population: assessment of an existing nomogram and a new predictive nomogram. Breast Cancer Res Treat 2012;135:839-48.

10. Kataria K, Srivastava A, Qaiser D. What Is a False Negative Sentinel Node Biopsy: Definition, Reasons and Ways to Minimize It. Ind J Surg 2016:78:396-401.

11. Hao Y, Ren G, Yang W, Zheng W, Wu Y, Li W, Li X, Li Y, Guo X. Combination diagnosis with elastography strain ratio and molecular markers effectively improves the diagnosis rate of small breast cancer and lymph node metastasis. Quant Imaging Med Surg 2020;10:678-91.

12. Bedi DG, Krishnamurthy R, Krishnamurthy S, Edeiken BS, Le-Petross H, Fornage BD. Cortical morphologic features of axillary lymph nodes as a predictor of metastasis in breast cancer: in vitro sonographic study. AJR Am J Roentgenol 2008;191:646-52.

13. Farrell TP, Adams NC, Stenson M, Carroll PA, Griffin M, 
Connolly EM. The Z0011 Trial: Is this the end of axillary ultrasound in the pre-operative assessment of breast cancer patients? Eur Radiol 2015;25:2682-7.

14. Wen X, Yu X, Tian Y, Liu Z, Cheng W, Li H, Kang J, Wei T, Yuan S, Tian J. Quantitative shear wave elastography in primary invasive breast cancers, based on collagen-S100A4 pathology, indicates axillary lymph node metastasis. Quant Imaging Med Surg 2020;10:624-33.

15. Yang J, Wang T, Yang L, Wang Y, Li H, Zhou X. Preoperative Prediction of Axillary Lymph Node Metastasis in Breast Cancer Using Mammography-Based Radiomics Method. Sci Rep 2019;9:4429.

16. Han L, Zhu Y, Liu Z, Yu T, He C, Jiang W. Radiomic nomogram for prediction of axillary lymph node metastasis in breast cancer. Eur Radiol 2019;29:3820-9.

17. Yu FH, Wang JX, Ye XH, Deng J, Hang J, Yang B. Ultrasound-based radiomics nomogram: A potential biomarker to predict axillary lymph node metastasis in early-stage invasive breast cancer. Eur J Radiol 2019;119:108658.

18. Hu HT, Wang Z, Huang XW, Chen SL, Zheng X, Ruan SM. Ultrasound-based radiomics score: a potential biomarker for the prediction of microvascular invasion in hepatocellular carcinoma. Eur Radiol 2019;29:2890-901.

19. Yi CB, Ding ZY, Deng J, Ye XH, Chen L, Zong M, Li CY. Combining the Ultrasound Features of Primary Tumor and Axillary Lymph Nodes Can Reduce False-Negative Rate during the Prediction of High Axillary Node Burden in BI-RADS Category 4 or 5 Breast Cancer Lesions. Ultrasound Med Biol 2020;46:1941-8.

20. Guo Q, Dong Z, Zhang L, Ning C, Li Z, Wang D, Liu C, Zhao M, Tian J. Ultrasound Features of Breast Cancer for
Predicting Axillary Lymph Node Metastasis. J Ultrasound Med 2018;37:1354-3.

21. Sopik V, Narod SA. The relationship between tumour size, nodal status and distant metastases: on the origins of breast cancer. Breast Cancer Res Treat 2018;170:647-56.

22. Koelliker SL, Chung MA, Mainiero MB, Steinhoff MM, Cady B. Axillary lymph nodes: US-guided fine-needle aspiration for initial staging of breast cancer--correlation with primary tumor size. Radiology 2008;246:81-9.

23. Hyun SJ, Kim EK, Moon HJ, Yoon JH, Kim MJ. Preoperative axillary lymph node evaluation in breast cancer patients by breast magnetic resonance imaging (MRI): Can breast MRI exclude advanced nodal disease? Eur Radiol 2016;26:3865-73.

24. Black D. Axillary Ultrasound: For All, for None, to Diagnose Positive Nodes, or to Support Avoiding Sentinel Lymph Node Biopsy Altogether. Ann Surg Oncol 2017;24:64-9.

25. Zhou LQ, Wu XL, Huang SY, Wu GG, Ye HR, Wei Q, Bao LY, Deng YB, Li XR, Cui XW, Dietrich CF. Lymph Node Metastasis Prediction from Primary Breast Cancer US Images Using Deep Learning. Radiology 2020;294:19-28.

26. Choi HY, Park M, Seo M, Song E, Shin SY, Sohn YM. Preoperative Axillary Lymph Node Evaluation in Breast Cancer: Current Issues and Literature Review. Ultrasound Q 2017;33:6-14.

27. Bevilacqua JL, Kattan MW, Fey JV, Cody HS 3rd, Borgen PI, Van Zee KJ. Doctor, what are my chances of having a positive sentinel node? A validated nomogram for risk estimation. J Clin Oncol 2007;25:3670-9.
Cite this article as: Jiang T, Su W, Zhao Y, Li Q, Huang P. Non-invasive prediction of lymph node status for patients with early-stage invasive breast cancer based on a morphological feature from ultrasound images. Quant Imaging Med Surg 2021;11(8):3399-3407. doi:10.21037/qims-20-1201 